\title{
Doping effect on the carrier scattering in iron-pnictide superconductors studied by charge transport
}

\author{
Shigeyuki Ishida ${ }^{\mathrm{a}, \mathrm{b}, \mathrm{e}}$, Masamichi Nakajima ${ }^{\mathrm{a}, \mathrm{b}, \mathrm{e}}$, Motoyuki Ishikado ${ }^{\mathrm{d}, \mathrm{e}}$, Yasuhide Tomioka ${ }^{\mathrm{b}, \mathrm{e}}$, Toshimitsu Ito ${ }^{\mathrm{b}, \mathrm{e}}$, Kiichi \\ Miyazawa $^{\text {b,c,e }}$, Chul-Ho Lee ${ }^{\text {b,e }}$, Hijiri Kito ${ }^{\text {,ee }}$, Shin-ichi Shamoto ${ }^{\text {d,e }}$, Akira Iyo ${ }^{\text {b,e }}$, Hiroshi Eisaki ${ }^{\text {b,e }}$, Kenji M. Kojima ${ }^{a, e}$, Teruhisa \\ Kakeshita $^{\mathrm{a}, \mathrm{e}}$, Shin-ichi Uchida ${ }^{\mathrm{a}, \mathrm{e}, *}$ \\ ${ }^{a}$ Department of Physics, University of Tokyo, Tokyo 113-0033, Japan \\ ${ }^{b}$ National Institute of Advanced Industrial Science and Technology, Tsukuba 305-8568, Japan \\ ${ }^{c}$ Department of Applied Electronics, Tokyo University of Science, Chiba 278-3510, Japan \\ ${ }^{d}$ Japan Atomic Energy Agency, Ibaraki, 319-1195, Japan \\ e JST, Transformative Research-Project on Iron Pnictides, Tokyo 102-0075, Japan
}

\begin{abstract}
In order to reveal the role of "carrier doping" in the iron-based superconductors, we investigated the transport properties of the oxygen-deficient iron-arsenides $L n \mathrm{FeAsO}_{1-y}(\mathrm{Ln}=\mathrm{La}, \mathrm{Ce}, \mathrm{Pr}$ and $\mathrm{Nd})$ over a wide doping range. We found that the effect of "doping" in this system is mainly on the carrier scattering rather than carrier density, quite distinct from that in high- $T_{\mathrm{c}}$ cuprates. In the case of La system with lower $T_{\mathrm{c}}$, the low temperature resistivity is dominated by $T^{2}$ term and fairly large magnetoresistance is observed. On the other hand, in the Nd system with higher $T_{\mathrm{c}}$, carriers are subject to stronger scattering showing nearly $T$-linear resistivity and small magnetoresistance. Such strong scattering appears intimately correlated with high- $T_{\mathrm{c}}$ superconductivity in the iron-based system.
\end{abstract}

Keywords:

superconductors, superconductivity, transport properties

PACS: 74.25.Fy, 74.62.Dh, 74.70.Dd

\section{Introduction}

The high- $T_{\mathrm{c}}$ cuprate and iron-based superconductors have several similarities. Among them, one important feature is that the parent compounds show antiferromagnetic order and superconductivity appears by doping carriers. In the case of high- $T_{\mathrm{c}}$ cuprates, carrier doping mainly controls carrier density and $T_{\mathrm{c}}$ is controlled by the superfluid density. On the other hand, in the iron-based superconductors, superconductivity can be induced through various methods; (1) electron doping ( $L n \mathrm{FeAsO}_{1-x} \mathrm{~F}_{x}[1,2], \mathrm{Ba}\left(\mathrm{Fe}_{1-x} \mathrm{Co}_{x}\right)_{2} \mathrm{As}_{2}$ [3], etc.), (2) hole doping $\left(\mathrm{Ba}_{1-x} \mathrm{~K}_{x} \mathrm{Fe}_{2} \mathrm{As}_{2}\right.$ [4], etc.), (3) isovalent doping $\left(\mathrm{BaFe}_{2}\left(\mathrm{As}_{1-x} \mathrm{P}_{x}\right)_{2}[5], \mathrm{Ba}\left(\mathrm{Fe}_{1-x} \mathrm{Ru}_{x}\right)_{2} \mathrm{As}_{2}\right.$ [6], etc.) and (4) applying pressure. As for latter two cases, carrier density is apparently unchanged. This indicates that carrier density is not so crucial for superconductivity in iron-based superconductors as in cuprates. Furthermore, it is empirically known that $T_{\mathrm{c}}$ of $\mathrm{LnFeAsO}$ system correlates strongly with the crystal structure and becomes maximum when FeAs 4 forms a nearly ideal tetrahedron [7]. However, it is still an unresolved puzzle why $T_{\mathrm{c}}$ is so different among different classes. In this work, we investigate the transport properties of the oxygen-deficient oxypnictides $L n \mathrm{FeAsO}_{1-y}(\mathrm{Ln}=\mathrm{La}, \mathrm{Ce}, \mathrm{Pr}$ and $\mathrm{Nd})$ to explore how

\footnotetext{
${ }^{*}$ Corresponding author.

Email address: uchida@phys.s.u-tokyo.ac.jp (Shin-ichi Uchida)
}

the charge transport in the normal state is correlated with the superconducting $T_{\mathrm{c}}$ in this class of pnictides.

\section{Experimental prodedures}

Polycrystalline samples of $L n \mathrm{FeAsO}_{1-y}$ were prepared by a high-pressure synthesis technique using a cubic-anvil-type apparatus (Riken CAP-07). [8] Well-sintered samples of high density, sufficient for transport measurements, were obtained by applying a pressure of $2 \mathrm{GPa}$ during the synthesis. Because some of the starting materials are quite reactive to oxygen, we could not prevent their oxidation in the synthesis procedure. The actual amount of oxygen deficiency $y$ was estimated with reference to the lattice constants [7] which were slightly smaller than those of the nominal composition. In order to make the distribution of the oxygen deficiency homogeneous, the samples were annealed in air at $450{ }^{\circ} \mathrm{C}$. A standard four-terminal method was used for the resistivity measurements. The Hall resistivity and the transverse MR were measured in a cryostat equipped with a superconducting magnet (PPMS, Quantum Design Inc.) with the magnetic field normal to the widest plane.

\section{Results and Discussion}

Figures 1 (a) - (d) display the temperature dependence of resistivity $\rho(T)$ for $\mathrm{LaFeAsO}_{1-y}, \mathrm{CeFeAsO}_{1-y}, \mathrm{PrFeAsO}_{1-y}$ and 

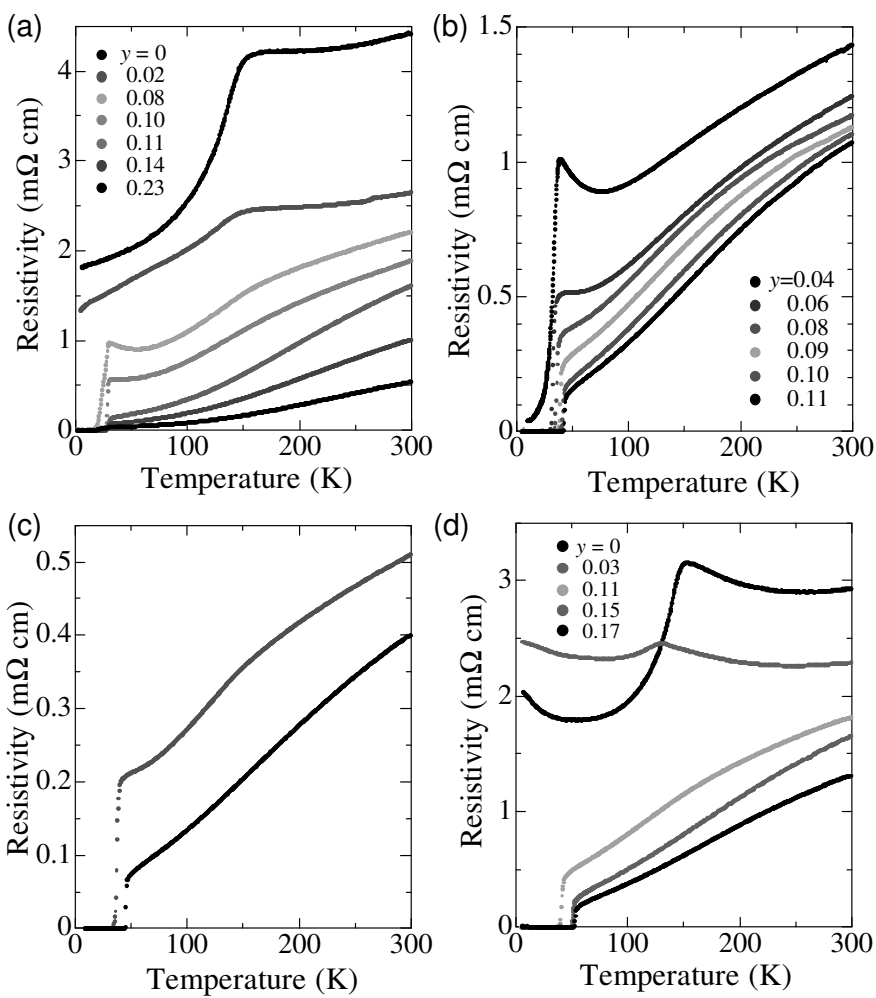

Figure 1: Temperature dependence of the resistivity of $\mathrm{LaFeAsO}_{1-y}$ for $y$ in the range $0 \leq y \leq 0.23$ (a), $\mathrm{CeFeAsO}_{1-y}$ for $y$ in the range $0.06 \leq y \leq 0.11$ (b), $\mathrm{PrFeAsO}_{1-y}$ from Ref. $10(\mathrm{c})$, and $\mathrm{NdFeAsO}_{1-y}$ for $y$ in the range $0 \leq y \leq 0.17$ (d).

$\mathrm{NdFeAsO}_{1-y}$, respectively. The evolution of $\rho(T)$ with $y$ looks qualitatively similar for all compounds. The most remarkable difference is that the normal-state resistivity in the superconducting regime is dominated by a $T^{2}$ term in $\mathrm{LaFeAsO}_{1-y}$, whereas by a $T$-linear term in $\mathrm{NdFeAsO}_{1-y}$. In addition, $\rho(T)$ of $\mathrm{CeFeAsO}_{1-y}$ and $\mathrm{PrFeAsO}_{1-y}$ seem to show intermediate behavior of those two.

To reveal the correlation between charge transport and $T_{\mathrm{c}}$, we investigated the exponent $n$ of $\rho(T) \sim T^{n}$ for each sample. We fitted the data in the form of $\rho(T)=\rho_{0}+A T^{n}$ in the temperature range between $T$ just above $T_{\mathrm{c}}$ and $T=150 \mathrm{~K}$, where $\rho_{0}$ is the residual component of resistivity. The exponent $n$ plotted against $T_{\mathrm{c}}$ is shown in Fig. $2 . \mathrm{NdFeAsO}_{1-y}$ with higher $T_{\mathrm{c}}$ clearly belongs to a class distinct from $\mathrm{LaFeAsO}_{1-y}$. On the same figure are also plotted the $n$-values for $\mathrm{CeFeAsO}_{1-y}$ and single crystals of $\mathrm{PrFeAsO}_{1-y}$ with $T_{\mathrm{c}}$ between 30 and $47 \mathrm{~K}$. We can see a crossover from $T^{2}$ to $T$-linear dependence of resistivity associated with an increase of $T_{\mathrm{c}}$. Then, one may conclude that the carrier scattering which gives rise to $T$-linear resistivity in the normal state is a possible ingredient for achieving $T_{\mathrm{c}}$ exceeding $50 \mathrm{~K}$ in iron-based superconductors.

Figure 3 shows magnetic field dependence of the transverse magnetoresistance (MR) for $\mathrm{LaFeAsO}_{1-y}, \mathrm{PrFeAsO}_{1-y}$ and $\mathrm{NdFeAsO}_{1-y}$ for $y=0.23,0.22$ and 0.17 , respectively. Note that carrier density is expected to be almost same for each compound. The magnitude of MR, which provides a measure of carrier mobility assuming a two-carrier model, is fairly large in

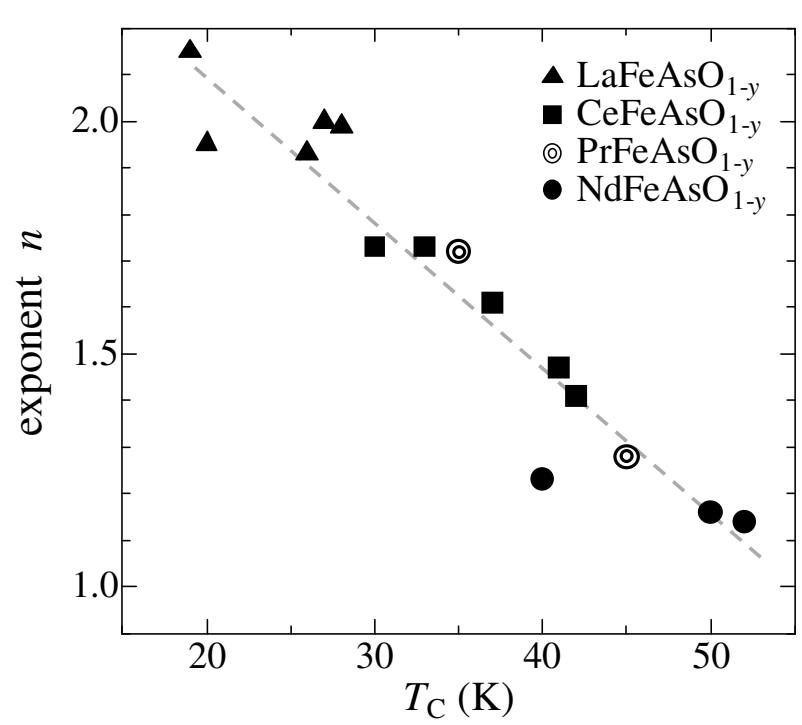

Figure 2: Relationship between $T_{\mathrm{c}}$ and the exponent $n$ of $\rho(T) \sim T^{n}$ for $L n \mathrm{FeAsO}_{1-y}(\mathrm{Ln}=\mathrm{La}, \mathrm{Ce}, \mathrm{Pr}$ and $\mathrm{Nd})$.

$\mathrm{LaFeAsO}_{1-y}(\sim 8 \%$ at $H=6 \mathrm{~T}$ and $T=50 \mathrm{~K})$. In contrast, MR is much smaller in $\mathrm{NdFeAsO}_{1-y}(\sim 1 \%$ at $H=6 \mathrm{~T}$ and $T=$ $70 \mathrm{~K})$. As for $\mathrm{PrFeAsO}_{1-y}$, MR takes an intermediate value between $\mathrm{LaFeAsO}_{1-y}$ and $\mathrm{NdFeAsO}_{1-y}(\sim 2 \%$ at $H=6 \mathrm{~T}$ and $T$ $=50 \mathrm{~K}$ ). Thus, carrier scattering (mobility) seems to be getting stronger (higher) by replacing $L n$ from $\mathrm{La}$ to $\mathrm{Nd}$.

In the band structure of nondoped $\mathrm{LaFeAsO}$ [11], the top of a hole band (called a $\gamma$ band) centered at $\Gamma$ is located just above the Fermi level. It moves down with electron doping and finally is located below the Fermi surface. In the case of $\mathrm{NdFeAsO}_{1-y}$ the top of the $\gamma$ band is higher than that for $\mathrm{LaFeAsO}_{1-y}$ being always above the Fermi level regardless of electron doping level. It is argued that a Fermi surface derived from the $\gamma$ band facilitates nesting between disconnected portions of electron Fermi surfaces, which would enhance "interband" scattering of electrons between the two nested Fermi surfaces and, in certain circumstances (e.g., when coupled with spin fluctuations), would be a source of pairing interaction $[12,13]$. In addition, the location of the $\gamma$ band is found to be sensitive to local crystal structure, specifically, the As height from the $\mathrm{Fe}$ plane or the bond angle $\alpha$ of the $\mathrm{FeAs}_{4}$ octahedron [11]. Then, the $\gamma$-Fermi surface seems to bridge between the known $T_{\mathrm{c}}-\alpha$ correlation and the present $T_{\mathrm{c}}-n$ correlation we observed.

\section{Conclusion}

The present results strongly suggest that the strong carrier scattering which gives rise to $T$-linear resistivity is intimately correlated with high- $T_{\mathrm{c}}$ in the Fe-based superconductors. "Doping" is not a parameter controlling carrier density but also carrier scattering. However, in the case of cuprates, the carrier scattering rate is not necessarily related with $T_{\mathrm{c}} . T_{\mathrm{c}}$ is controlled by the superfluid density, and the transport scattering rate is not strongly dependent on doping and material in the underdoped and optimally doped regimes. In view of the charge transport 


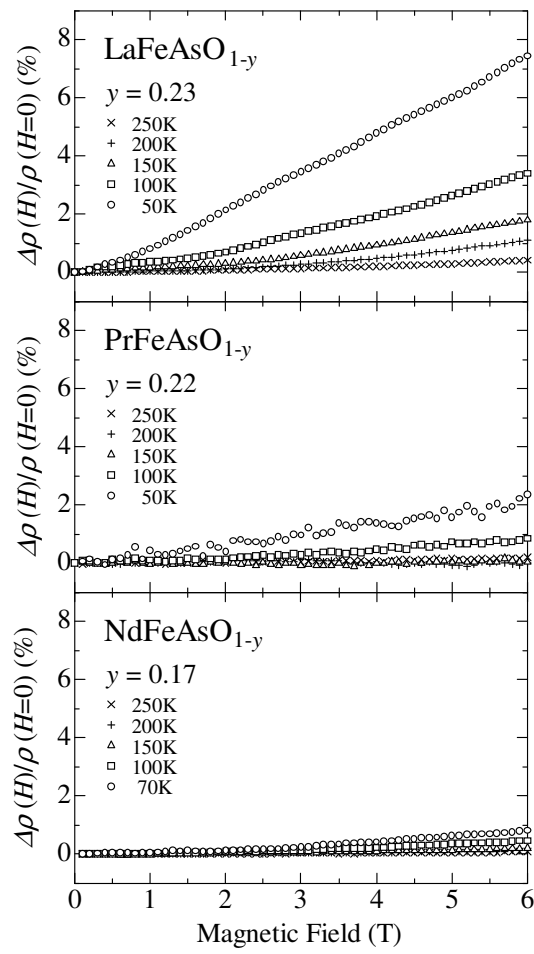

Figure 3: Magnetic field dependence of the transverse magnetoresistance of $\mathrm{LaFeAsO}_{1-y}, \mathrm{PrFeAsO}_{1-y}$ and $\mathrm{NdFeAsO}_{1-y}$ for $y=0.23,0.22$ and 0.17 , respectively.

demonstrated in the present work, the Fe-based superconductors apparently belong to a class different from the cuprates, and seem to have an aspect in common with other classes of superconductors in that the carrier scattering has some relevance to $T_{\mathrm{c}}$.

\section{Acknowledgment}

We would like to acknowledge K. Kuroki, H. Aoki, S. Ishibashi, and T. Miyake for helpful discussions. This work was supported by the Global Centers of Excellence Program and A3 Foresight Program from the Japan Society for the Promotion of Science and Transformative Research-Project on Iron Pnictides from the Japan Science and Technology Agency.

[1] Y. Kamihara, T. Watanabe, M. Hirano, H. Hosono, J. Am. Chem. Soc. 130, (2008) 3296

[2] X. H. Chen, T. Wu, G. Wu, R. H. Liu, H. Chen, D. F. Fang, Nature 453, (2008) 761

[3] A. S. Sefat, R. Jin, M. A. McGuire, B. C. Sales, D. J. Singh, D. Mandrus, Phys. Rev. Lett. 101, (2008) 117004

[4] M. Rotter, M. Tegel, D. Johrendt, Phys. Rev. Lett. 101, (2008) 107006

[5] S. Jiang, H. Xing, G. Xuan, C. Wang, Z. Ren, C. Feng, J. Dai, Z. Xu, G. Cao, J. Phys.: Condens. Matter 21, (2009) 382203

[6] S. Sharma, A. Bharathi, S. Chandra, V. R. Reddy, S. Paulraj, A. T. Satya, V. S. Sastry, A. Gupta, and C. S. Sundar, Phys. Rev. B 81, (2010) 174512

[7] C. H. Lee, A. Iyo, H. Eisaki, H. Kito, M. T. Fernandez-Diaz, T. Ito, K. Kihou, H. Matsuhata, M. Braden, K. Yamada, J. Phys. Soc. Jpn. 77, (2008) 083704

[8] H. Kito, H. Eisaki, A. Iyo, J. Phys. Soc. Jpn. 77, (2008) 63707

[9] Y. Jia, P. Cheng, L. Fang, H. Luo, H. Yang, C. Ren, L. Shan, C. Gu, H. H. Wen, Appl. Phys. Lett. 93, (2008) 032503

[10] M. Ishikado, S. Shamoto, H. Kito, A. Iyo, H. Eisaki, T. Ito, Y. Tomioka, Physica C 469, (2009) 901 / M. Ishikado, et al., unpublished
[11] K. Kuroki, H. Usui, S. Onari, R. Arita, H. Aoki, Phys. Rev. B 79, (2009) 224511

[12] I. I. Mazin, D. J. Singh, M. D. Johannes, M. H. Du, Phys. Rev. Lett. 101, (2008) 057003

[13] K. Kuroki, S. Onari, R. Arita, H. Usui, Y. Tanaka, H. Kontani, H. Aoki, Phys. Rev. Lett. 101, (2008) 087004 